1 Fachada hacia el oriente, calle Froilán Roa; a la izquierd parte del edificio de estacionamientos origin 2 Hall $5^{\circ}$ nivel, vista hacia el oriente

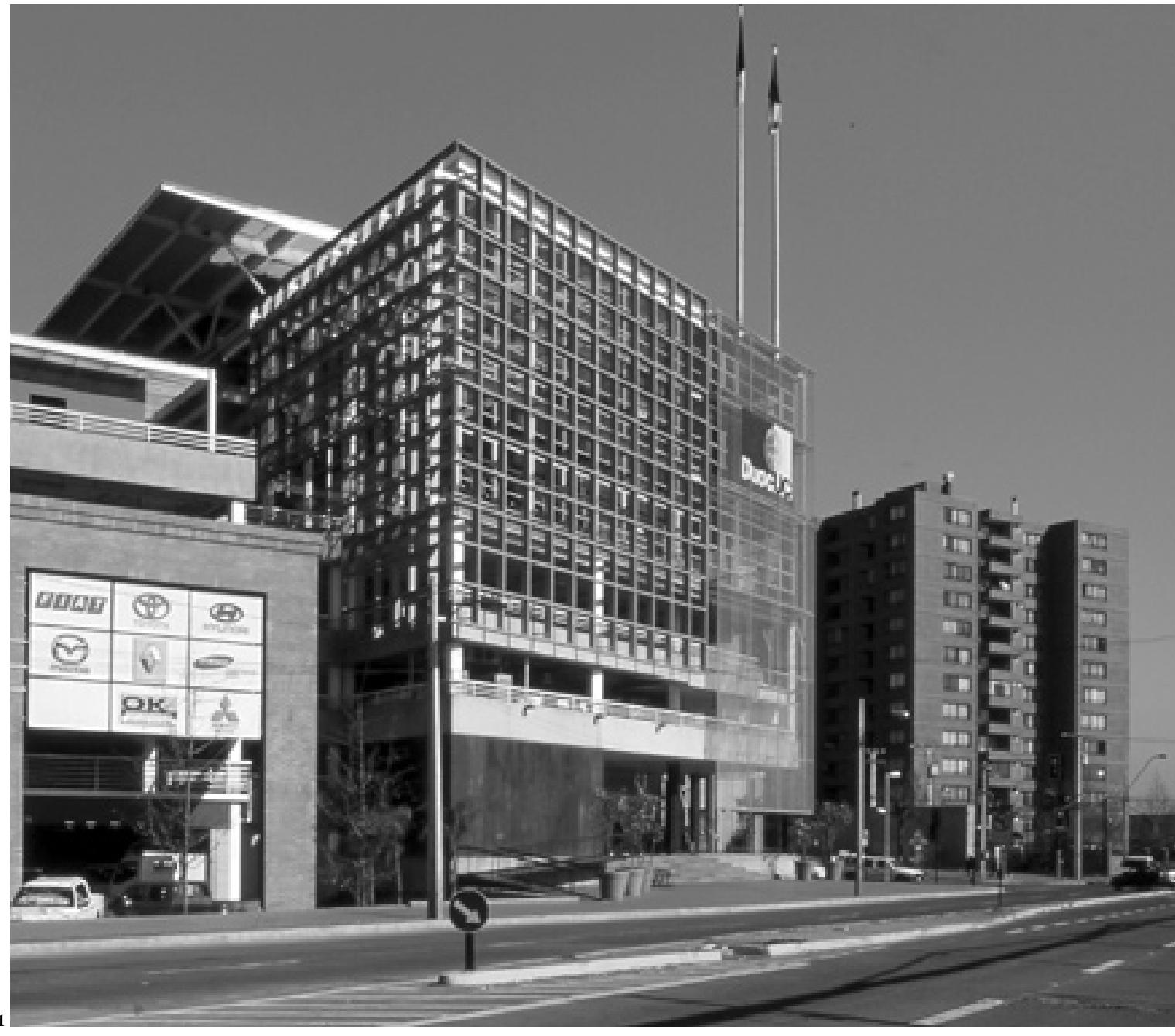

\title{
Duoc U.C. La Florida
}

La Florida, Chile

¿La periferia no es urbana? El edificio del DUOC en La Florida, instalado sobre un edificio de estacionamientos junto al mall con mayor volumen de ventas en Santiago, desmonta muchos supuestos asociados a los nuevos barrios. En un contexto denso, hibrido y dinámico, donde conviven peatones, automóviles, multitiendas, torres de departamentos y centros para el ocio, el proyecto toma el pulso de esta realidad con decisiones que apuntan a la flexibilidad y el intercambio. Su emplazamiento, una lógica constructiva desmontable y un piso noble en la azotea señalan una dirección para una posible urbanidad contemporánea.

Palabras clave: Arquitectura - Chile, establecimientos educacionales, educación técnica superior, centros urbanos.

So the outskirts are not city, are not urbane? DUOC's building in La Florida, built over the parking lot of the biggest-selling mall in Santiago, breaks with many assumptions about the new neighborboods. In a dense, bybrid, fast-changing setting, a place where pedestrians, cars, department stores, apartment blocks and leisure centers rub shoulders, the project takes its measure and comes up with solutions that aim at flexibility and inter-change. The location, dismountable construction, and the keynote final floor rising from the flat roof suggest a possible way forward for modern-day city living.

Key words: Architecture - Chile, educational institutions, higher
Duoc es una Fundación Educacional de la Universidad Católica que por más de 30 años orienta su labor a la educación técnicoprofesional, en un país cuya educación superior está fuertemente orientada a carreras universitarias. Dado el carácter y alcance de su labor, la mayoría de los estudiantes provienen de sectores medios y bajos, sin embargo el prestigio de la Institución y la calidad de la enseñanza impartida permiten un alumnado con un promedio de puntajes de ingreso similar a la media nacional universitaria.

La Fundación se financia con recursos provenientes de matrículas, donaciones y venta de servicios de capacitación para trabajadores y técnicos, reinvirtiendo sus excedentes en el mejoramiento de su infraestructura y nivel docente.
La puesta en valor del Duoc, cuyo principal objetivo es la búsqueda de la excelencia académica y la formación humana de sus estudiantes, otorga a las sedes un rol emblemático en la dignificación de los espacios para la educación y su directa relación en la formación de profesionales de alto nivel. La calidad de la arquitectura no es una consecuencia, sino un segundo objetivo en la definición de una espacialidad que acoja y favorezca el proyecto académico.

El encargo debía, a través de la arquitectura, dar respuesta a una asociación entre Duoc UC y el mall Plaza Vespucio. En una fusión de intereses, nace la idea de proyectar un edificio que forme técnicos y profesionales orientados al área comercial, nicho que hoy en Chile constituye el $20 \%$ de la fuerza laboral del país.

Proponiéndose como terreno el piso superior 


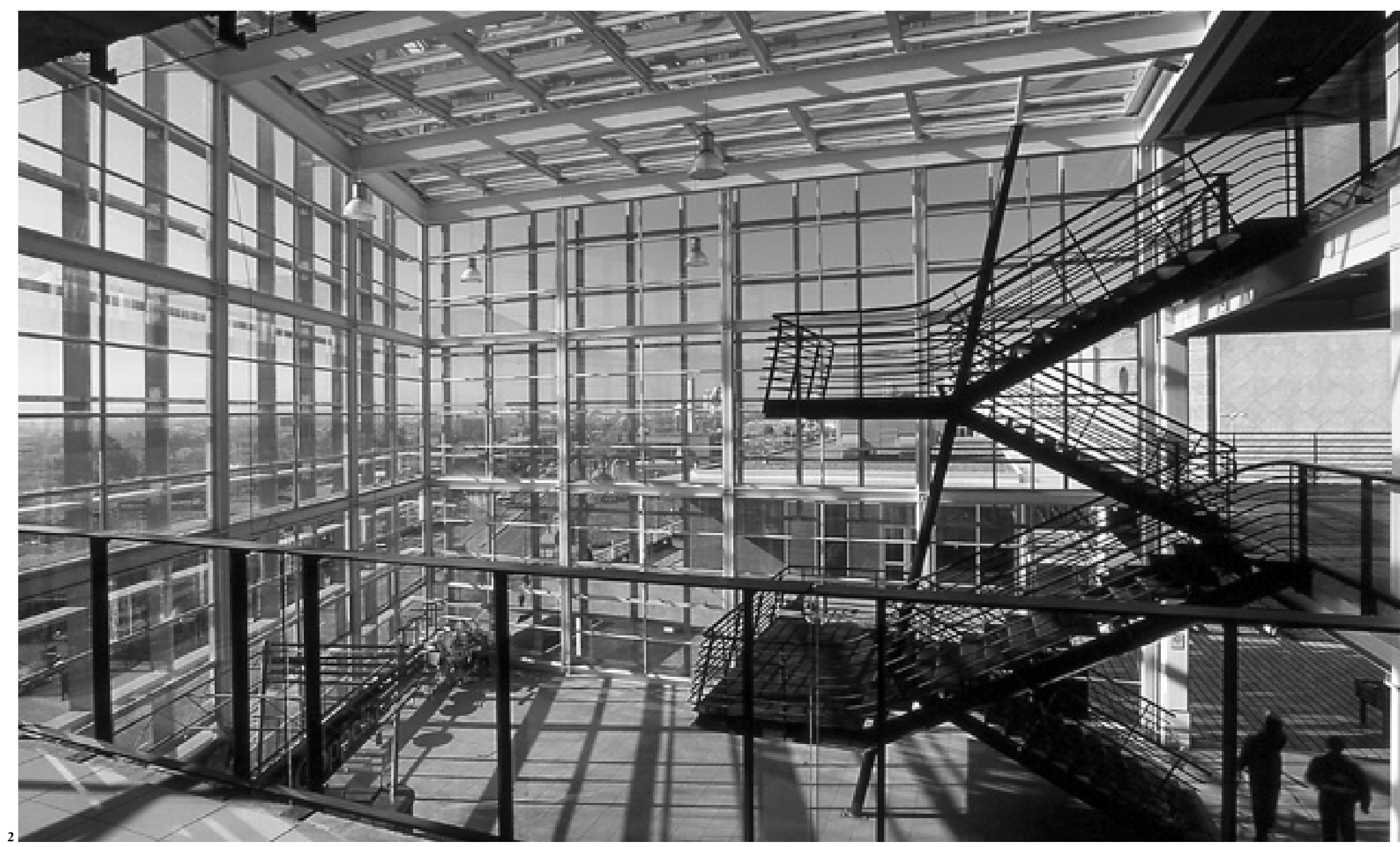

de un edificio de estacionamientos en la esquina nor-oriente del mall, el tema giraría en torno a la posesión e imagen del edificio en un entorno inmediato de carácter comercial. Se parte de la solución de dos premisas iniciales: organizar la estructura y el programa del edificio a través de un módulo y una geometría dada, que correspondía al módulo $8 \times 8 \mathrm{~m}$ en estructura de acero (propio del park deck), y resolver el acceso desde la calle hasta el nivel destinado al proyecto en la losa superior de los estacionamientos, cuatro niveles más arriba.

El edificio se organiza en la cubierta del park deck, construyendo los bordes hacia las vistas principales del valle de Santiago y la cordillera. Hacia el interior una plaza definida por dos bloques paralelos de tres niveles, con salas de clases y recintos comunes, y un bloque perpendicular de un nivel con el programa administrativo.
La plaza, delimitada en sus dos costados por los volúmenes, se diseñó como un espacio de recreación y expresión para los alumnos, patio de estudio, ocio, reunión y actividades.

Estos dos bloques paralelos de tres niveles se relacionan inversamente entre sí: el bloque que conforma el borde exterior define las transparencias de sus recintos hacia la calle y sus circulaciones y accesos a salas de clase hacia el interior. El segundo bloque dispone sus transparencias hacia el interior y sus circulaciones y accesos hacia la plaza; entre éstos se desarrolla un espacio lineal cubierto de tres niveles de altura que remata en el hall principal vidriado.

La conexión vertical se muestra en la fachada a través de un sistema de escaleras mecánicas que recorren los niveles intermedios del park deck.

La imagen del proyecto busca, a través de la transparencia, contrastar con la hermeticidad del mall, presentando a la ciudad una fachada viva que permite ver los actos acogidos en su interior, haciendo de la educación una actividad que otorga dinamismo y actualidad a la ciudad. Sustentado esto, además, en el lenguaje arquitectónico contemporáneo, la cualificación de la luz al interior y una vinculación visual al contexto urbano inmediato desde los lugares públicos del edificio.

Una malla de metal desplegado que recorre libremente la fachada del edificio y estacionamientos, vincula los niveles superiores del proyecto a los del park deck con una envolvente permeable que unifica los distintos roles de un edificio en el que convive una multiplicidad de situaciones. Todo ello busca orientar a la educación hacia esferas más allá del aula típica donde se imparten las clases, democratizando y exponiendo su entrega. ARQ 

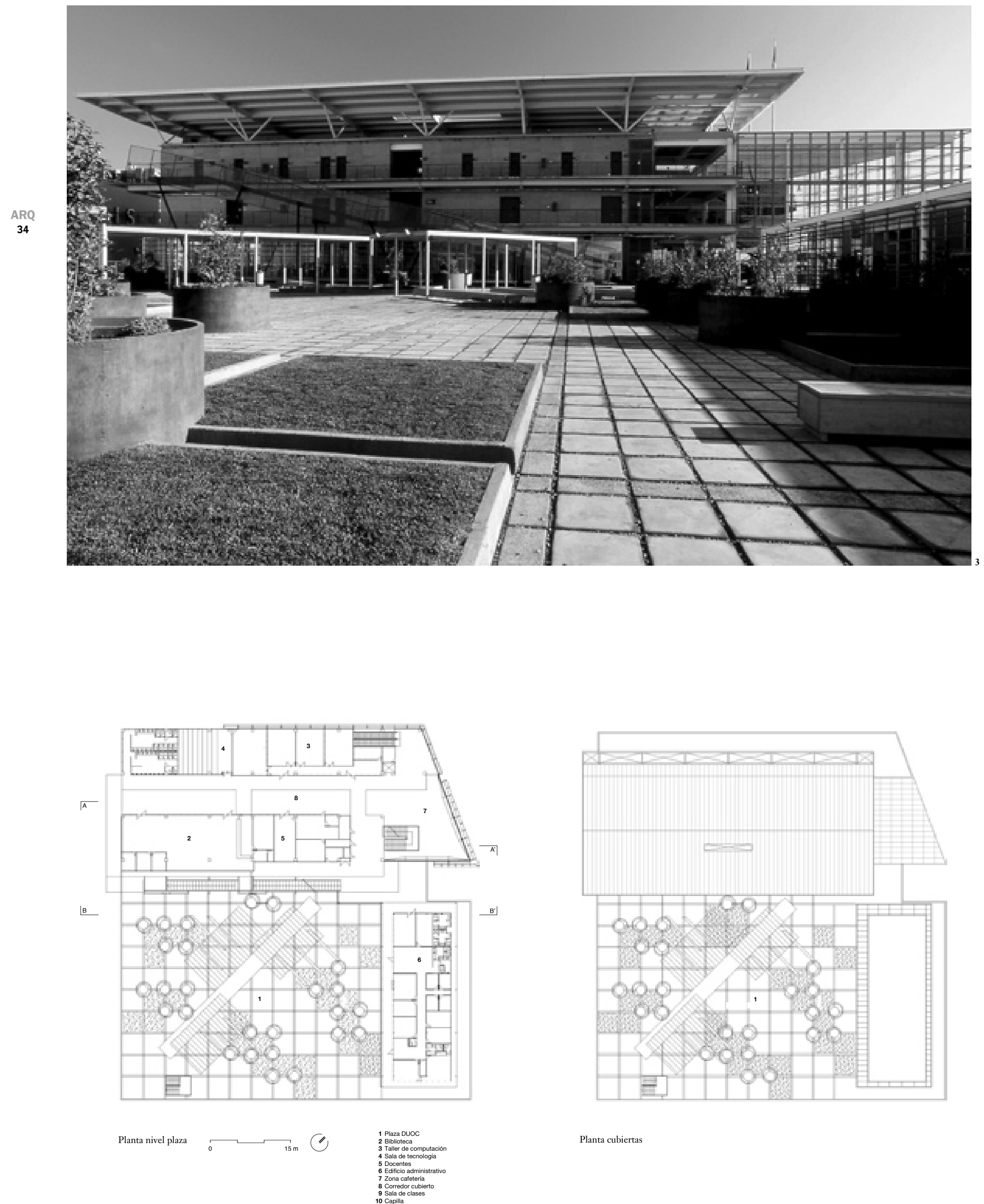
3 La plaza en la cubierta del al fondo, el bloque sur de aula

4 Vista desde la plaza, bloque de aulas a la izquierda y bloque $\mathrm{d}$ administración a la derecha
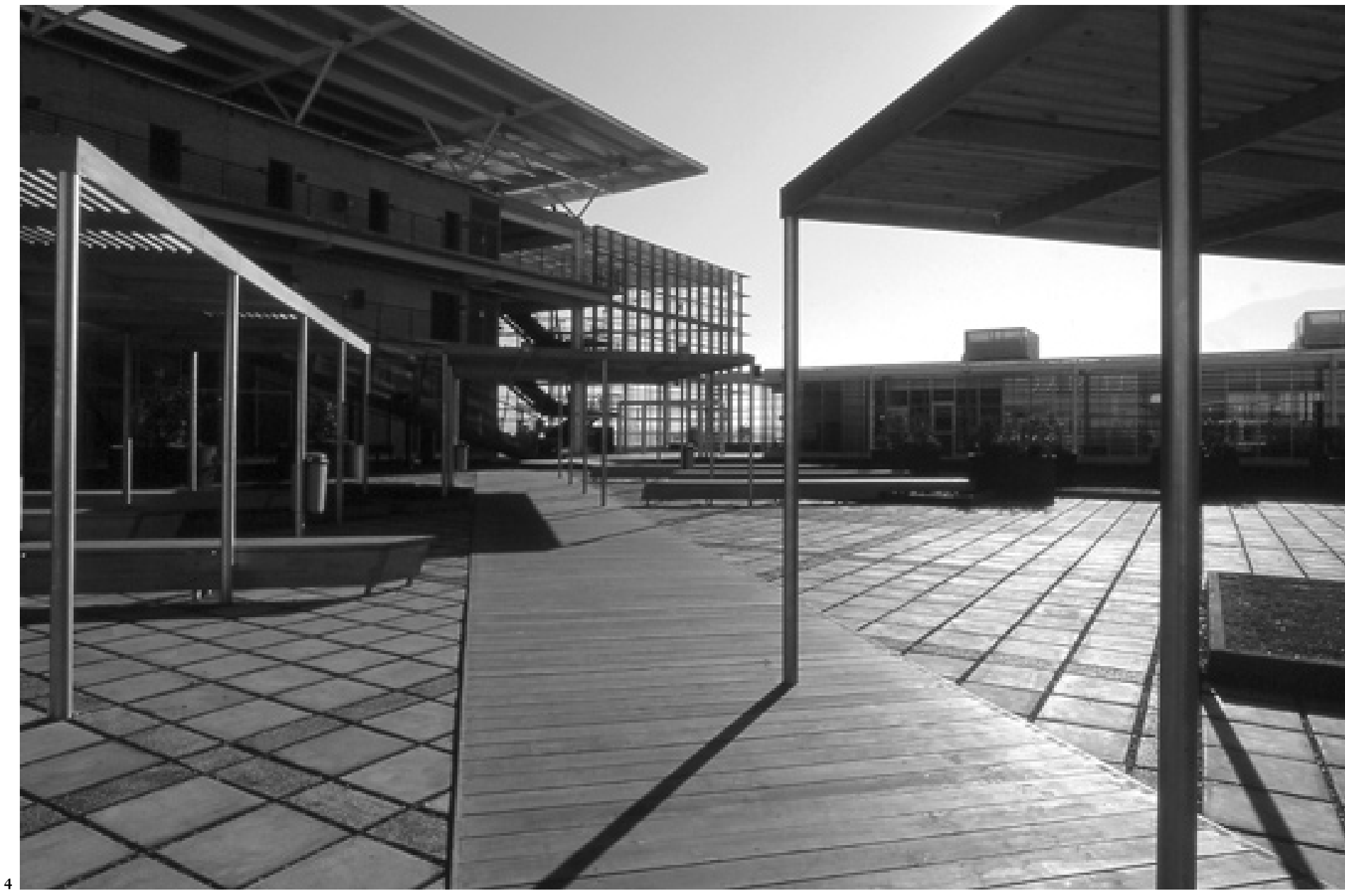

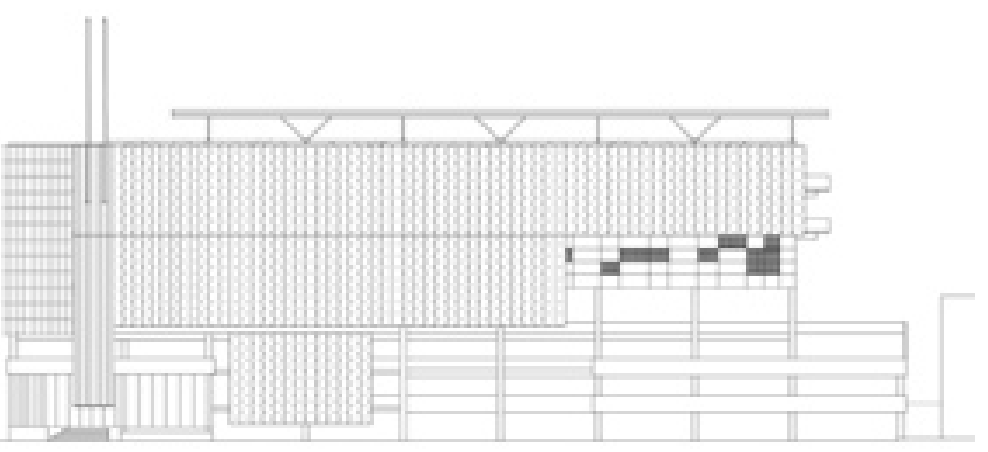

B

Planta piso tipo, bloques de aulas y espacios comunes

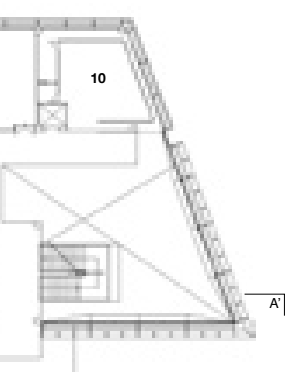

B!

Elevación nor-poniente

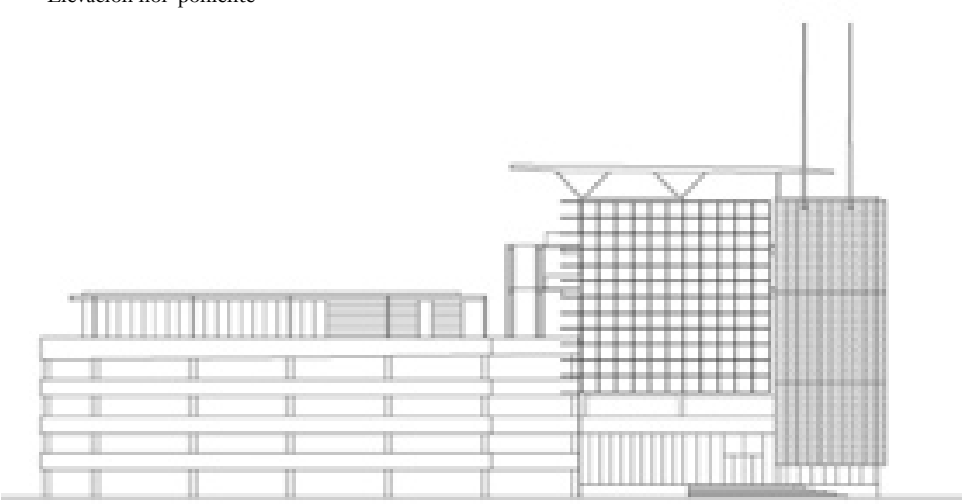



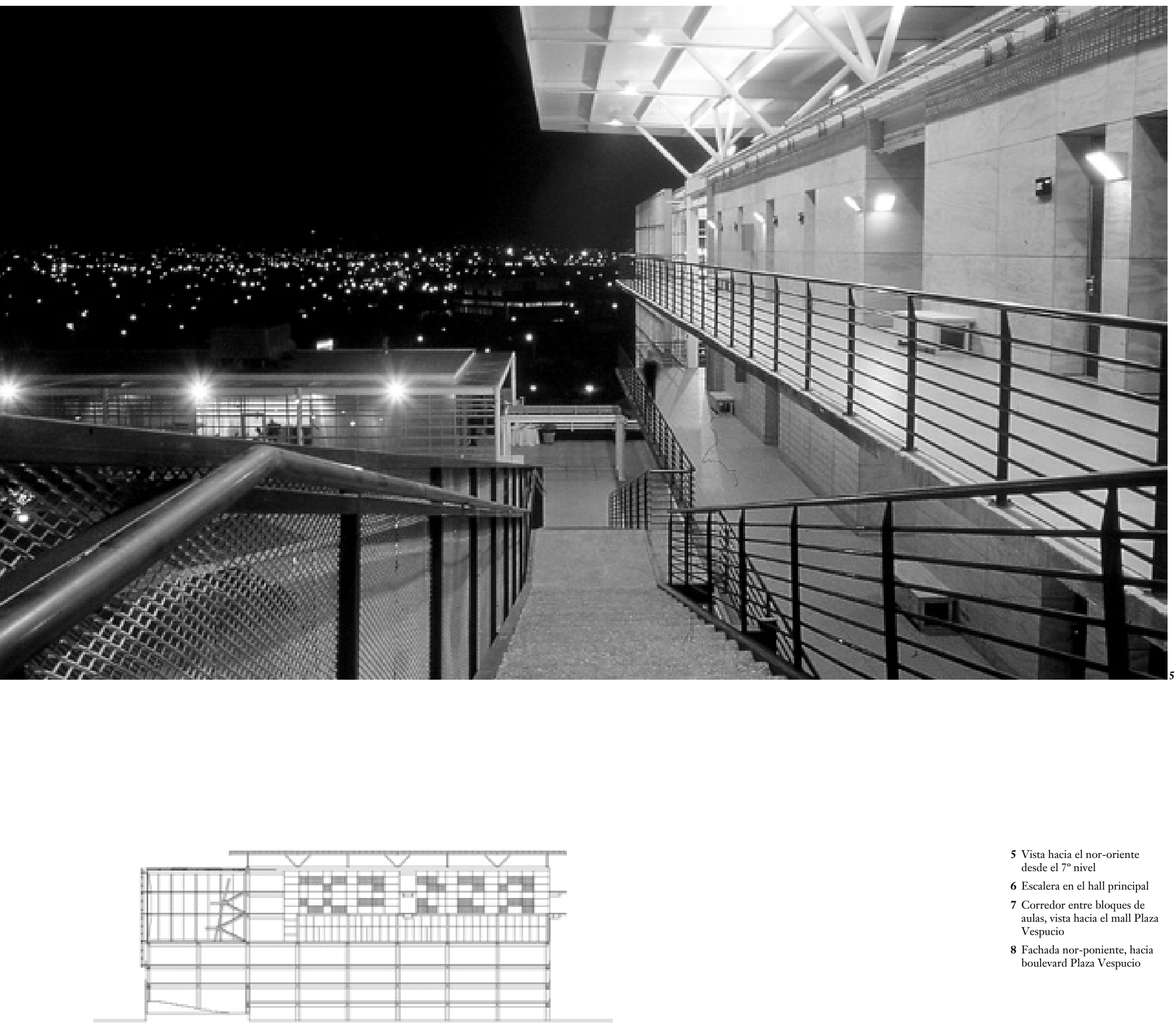

5 Vista hacia el nor-oriente

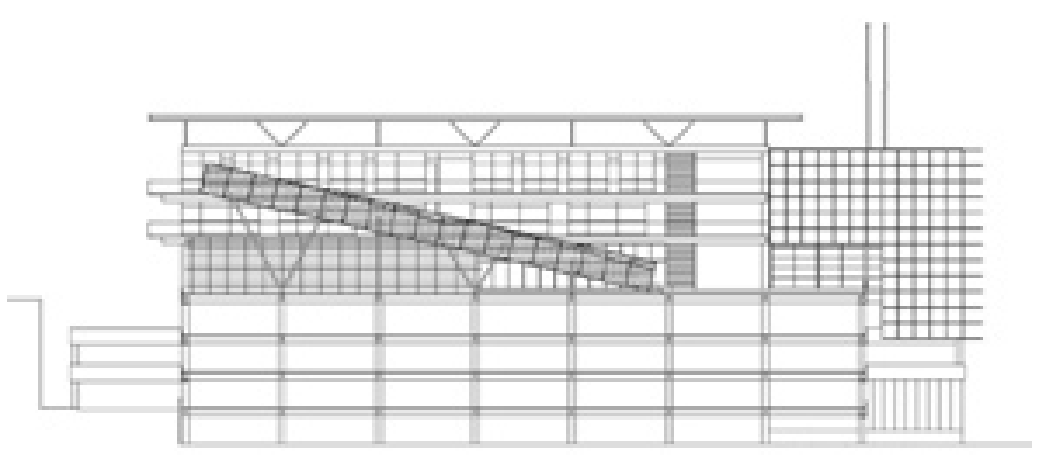




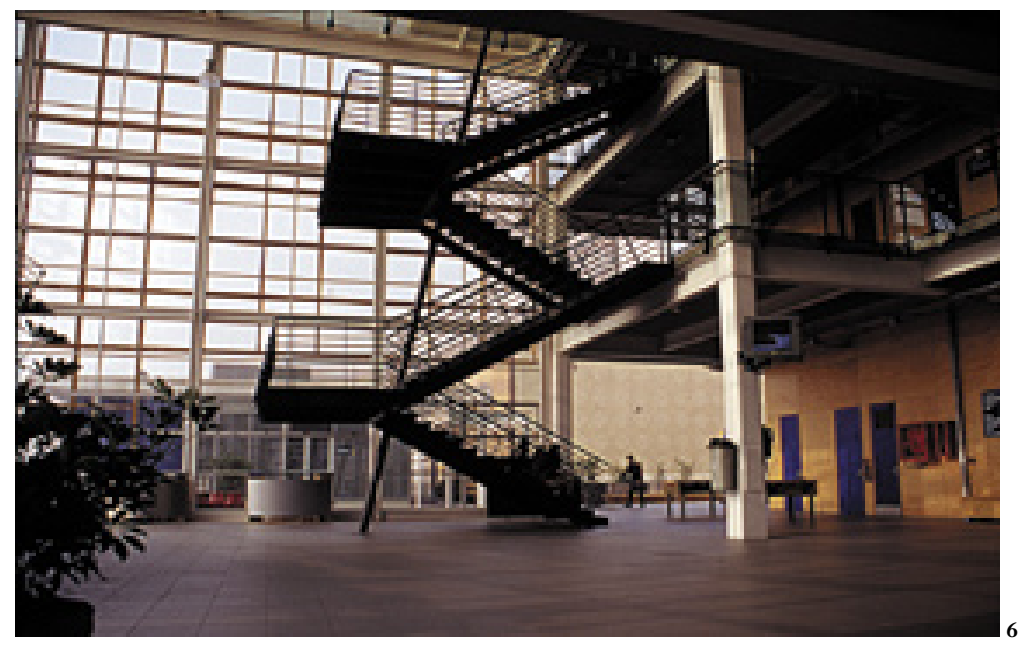

Duoc U.C. La Florid

Arquitectos Juan Sabbagh, Mariana Sabbagh, Marcial Olivares

y Juan Pedro Sabbagh

Colaboradores Felipe Sabbash y Cristóbal Noguera

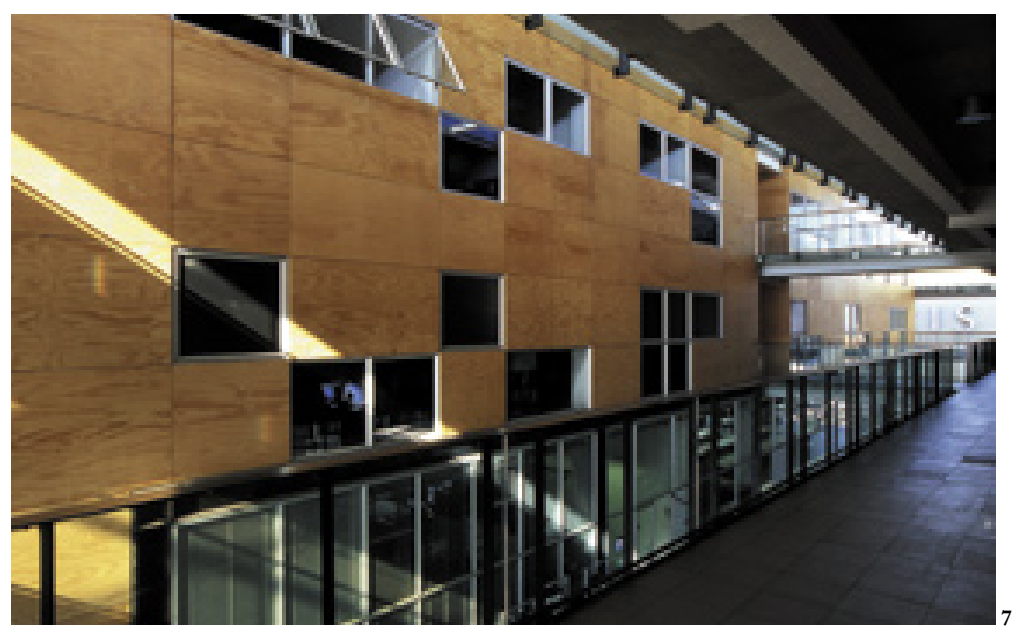

Ubicación Calle Froilán Roa esq. Boulevard Plaza Vespucio, La

Florida, Santiago, Chile

Cliente Fundación Duoc Universidad Católica

Cálculo estructural Arce Rezine Asociados

Construcción Constructora de Mussy

Instalaciones sanitarias Giaretti Hnos.

Proyecto eléctrico y corrientes débiles Ipel Ltda.

Iluminación Mónica Pérez

Climatización Gormaz y Zenteno

Materialidad Estructura de acero, cerramientos exteriores

de malla de acero y cristal, particiones interiores en áreas

públicas de cristal y madera, celosías de madera

Presupuesto 17,2 UF/ m² (US\$ 418/ $\mathrm{m}^{2}$ )

Superficie terreno $4.200 \mathrm{~m}^{2}$

Superficie construida $4.700 \mathrm{~m}^{2}$

Año proyecto 2002

Año construcción 2002 - 2003

Fotografía Guy Wenborne (diurnas) y Francisca Polanco (nocturnas)

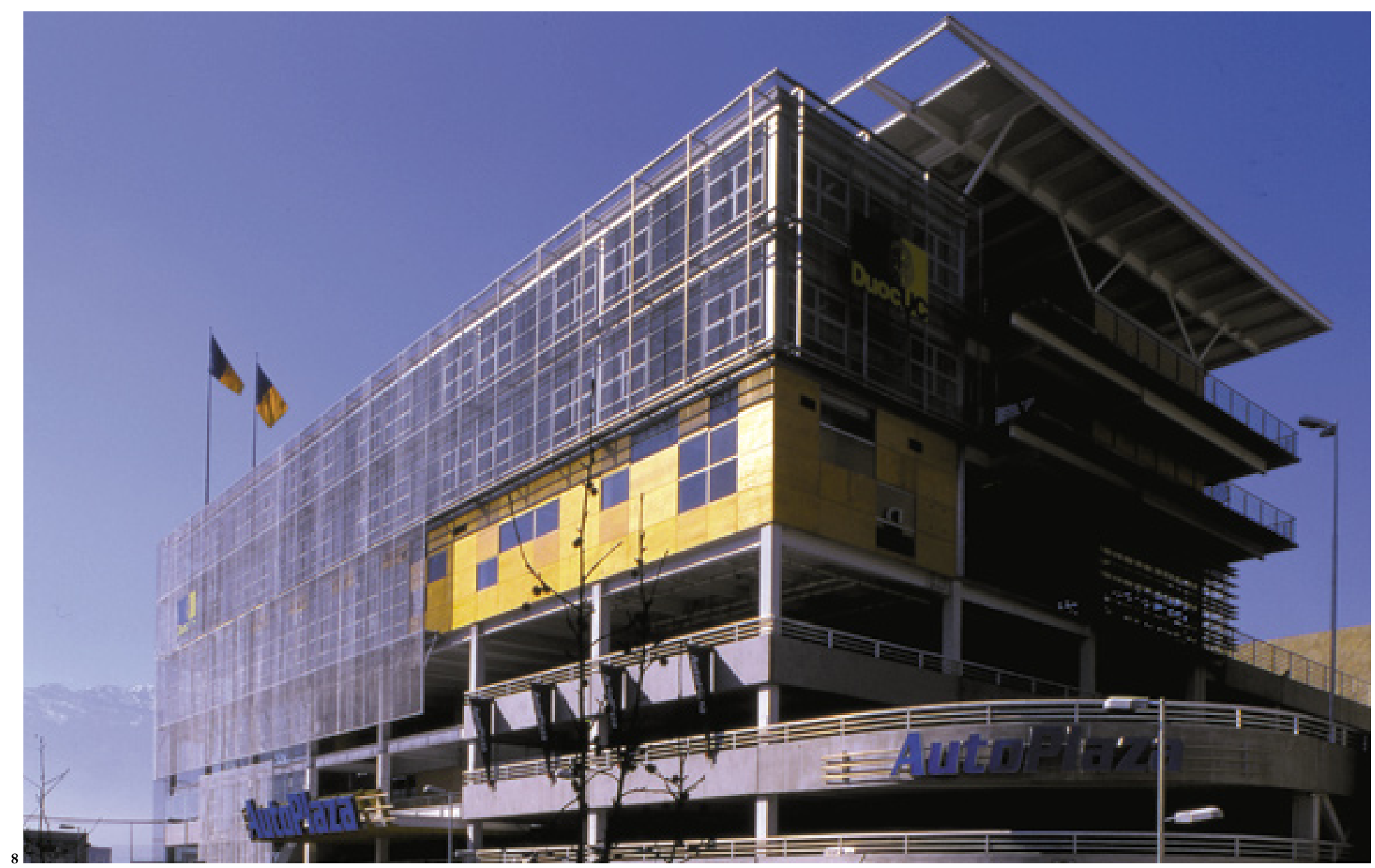

